\title{
A Mechatronic Platform for Computer Aided Detection of Nodules in Anatomopathological Analyses via Stiffness and Ultrasound Measurements
}

\author{
Luca Massari $^{1, *}$, Andrea Bulletti ${ }^{2,+}\left(\mathbb{D}\right.$, Sahana Prasanna ${ }^{1,+}+$, Marina Mazzoni ${ }^{2,3}(\mathbb{D}$, \\ Francesco Frosini ${ }^{4}$, Elena Vicari ${ }^{1}$, Marcello Pantano ${ }^{1}$, Fabio Staderini ${ }^{4}$, Gastone Ciuti ${ }^{1}$, \\ Fabio Cianchi ${ }^{4}$, Luca Messerini ${ }^{4}$, Lorenzo Capineri ${ }^{2}{ }^{\mathbb{D}}$, Arianna Menciassi ${ }^{1}$ and \\ Calogero Maria Oddo $1, *$ (D) \\ 1 Sant'Anna School of Advanced Studies, The BioRobotics Institute, 56025 Pisa, Italy; \\ sahana.prasanna@santannapisa.it (S.P.); elenavicari.ev@gmail.com (E.V.); \\ marcello.pantano03@gmail.com (M.P.); gastone.ciuti@santannapisa.it (G.C.); \\ arianna.menciassi@santannapisa.it (A.M.) \\ 2 Department of Information Engineering, Università Degli Studi di Firenze, 50139 Florence, Italy; \\ andrea.bulletti@unifi.it (A.B.); m.mazzoni@ifac.cnr.it (M.M.); lorenzo.capineri@unifi.it (L.C.) \\ 3 Consiglio Nazionale delle Ricerche of Italy, Istituto di Fisica Applicata "Nello Carrara", 50121 Florence, Italy \\ 4 Azienda Ospedaliera Careggi University Hospital of Florence and University of Florence, 50134 Florence, \\ Italy; francesco.frosini@unifi.it (F.F.); staderini.fabio@gmail.com (F.S.); fabio.cianchi@unifi.it (F.C.); \\ luca.messerini@unifi.it (L.M.) \\ * Correspondence: luca.massari@santannapisa.it (L.M.); calogero.oddo@santannapisa.it (C.M.O.) \\ + These authors share second authorship based on equal contribution.
}

Received: 30 March 2019; Accepted: 29 May 2019; Published: 31 May 2019

\begin{abstract}
This study presents a platform for ex-vivo detection of cancer nodules, addressing automation of medical diagnoses in surgery and associated histological analyses. The proposed approach takes advantage of the property of cancer to alter the mechanical and acoustical properties of tissues, because of changes in stiffness and density. A force sensor and an ultrasound probe were combined to detect such alterations during force-regulated indentations. To explore the specimens, regardless of their orientation and shape, a scanned area of the test sample was defined using shape recognition applying optical background subtraction to the images captured by a camera. The motorized platform was validated using seven phantom tissues, simulating the mechanical and acoustical properties of ex-vivo diseased tissues, including stiffer nodules that can be encountered in pathological conditions during histological analyses. Results demonstrated the platform's ability to automatically explore and identify the inclusions in the phantom. Overall, the system was able to correctly identify up to $90.3 \%$ of the inclusions by means of stiffness in combination with ultrasound measurements, paving pathways towards robotic palpation during intraoperative examinations.
\end{abstract}

Keywords: cancer nodules detection; phantom; stiffness analysis; ultrasound analysis; visual analysis; automatic robotic platform; remote support for pathologists

\section{Introduction}

Cancer is an abnormal and uncontrolled cell growth that invades healthy tissues, and that can spread via metastases to other locations in the body [1]. Various cancer treatments involve chemical and radiation therapies or surgery [2-4]. Following surgical intervention, biopsy is performed on the lymph nodes excised from the tissue to properly characterize cancer spread and examine whether it has developed the ability to spread to other lymph nodules or organs too. The accuracy in estimating 
the amount of spread of cancer is extremely important to avoid complications caused by an extensive resection of healthy lymph nodes and tissues. Accurate localization of tumors in tissues resected during surgery can also allow the surgeon to decide and modify in itinere the planned intervention so to remove malignant tissues missed in pre-operative imaging. Stiffness of human tissue is higher for tumor nodules with respect to healthy tissues [5-10]. Hence, inspecting the mechanical properties of cancerous tissues can contribute to the detection of nodules. Intraoperative palpations of the resected malignant tissue provide essential information about the presence of abnormalities [11]. Indeed, such investigation is part of the general practice performed by a specialist through manual palpation to retrieve several information about cancer nodules [12]. The reliable confidence of medical practitioners to detect tumors is achieved with rigorous training before they reach proper expertise in examining various organs and detecting abnormalities [13]. The human capability to detect lumps in the tissues, however, degrades with increasing lump depth, decreased compliance of the tissue, deformation of the finger pad induced by the lump itself, and the finger indentation velocity [14,15]. Ultrasound analysis [16] can complement stiffness data because of the different acoustic properties of cancer nodules, as demonstrated by intraoperative ultrasonography recordings having reported influence (varying from $2.7 \%$ up to $73 \%$ ) on the surgical procedures that were preoperatively planned [17-20]. Elastography is also used for the investigation of many diseases in tissues (liver, breast, thyroid, kidney, prostate, and lymph nodes) [21]. The elastographic equipment is of two types depending on the US sensors arrangement: Strain imaging and shear wave imaging. In both cases a US array probe is gel coupled to tissues; the latter requirement represents a limiting factor for the anatomo-pathology examinations for which any contamination of tissue samples must be avoided and only the natural wetness of excised tissues is allowed.

In this study, we combined stiffness and ultrasound data to aid the intraoperative histological exams performed on tissues excised during surgery by the identification of regions with different stiffness and US impedance characteristics. Such an examination is crucial in case of misdiagnosis or in case of unforeseeable diagnostic queries that might arise during surgery. Results from the examination may be used as a guide for surgical resection and decision-making to modify the common surgical procedure (see Figure 1). Initially, the surgeon gets a small tissue specimen $(2-3 \mathrm{~cm})$ from patient in order to get information about the presence of pathological patterns. Then, this specimen is placed on an automated system able to move and scan the material in order to get the data. The system proposed in this work is based on a precision mechanical scanner that provides a map of the tissue sample with inclusions. Data were derived from a sensor designed with a load cell for stiffness measurements, which also ensures the contact force on the sample to a vertically supported needle-type ultrasonic transducer provided for US measurements. In this way, two types of data (mechanical stiffness and US impedance) are measured at the same time in each position providing in practice two maps with well-correlated information regarding the positions of inclusions within the scanned area. Finally, with the position of the inclusion the pathology lab technician is able to sample the material and create a precise number of slices for microscopy analysis. Directly from the operating theatre the images are sent to the pathologist for the remote diagnosis. The time between the initial part of the process (excision) and the final part (medical report) should be contained within 20-30 min.

With instrumented tools, automatic classification of tumors in tissues can be addressed by machine learning techniques: Supervised-unsupervised classification, clustering and learnt neural networks $[22,23]$. The proposed system aims at reproducing the activity of pathologists in intraoperative tumor identification using feedback from vision, stiffness [24] and ultrasound measurements [25]. Using a robotic platform, and machine learning techniques for classification, the focus of this work is to detect and localize nodules buried in phantoms mimicking the elastic and ultrasound properties of excised human tissues. Specifically, the experimental evaluation was carried out by means of Agar-based phantoms suited to mimic liver, cardiac, brain and soft tissues [26-28], either in their acoustic and mechanical properties and temperature dependency $[29,30]$. 
The paper is organized as follows. Section 2 describes the experimental setup, the technical specifications of the used phantoms, the experimental protocol and data analysis methods. Results are presented in Section 3, showing the results of stiffness and ultrasound data analyses both separately and merging them together. The last section concludes with the discussion of the entire work and presents potential future investigations.

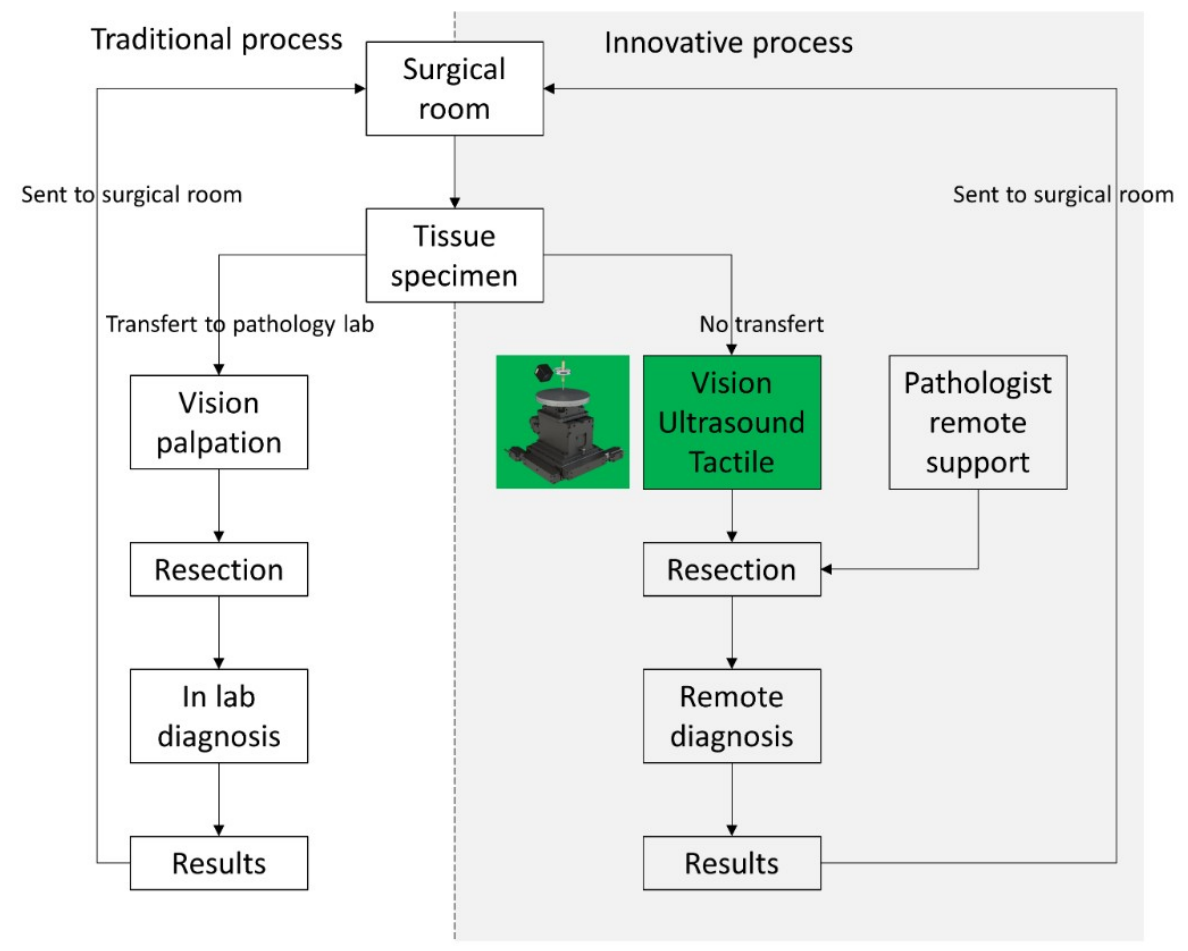

Figure 1. Block diagram of the histological procedure. On the left the traditional process is shown, whereas the proposed process is depicted on the right.

\section{Materials and Methods}

\subsection{Platform Design}

A platform was developed to detect embedded rigid inclusions surrounded by a soft matrix. The automated system consists of the following components (Figure 2):

(i) Three motorized translational stages and one rotational stage allowing to move the sample. A commercial stage (8MTF-102LS05, STANDA, Vilnius, Lithuania) with $10 \mathrm{~cm}$ of travel range and a resolution of $2.5 \mu \mathrm{m}$ was used for the $X$ and $Y$ axes, while another translational stage (8MVT120-25-4247, STANDA, Vilnius, Lithuania) was used to indent the sample along the $Z$ axis, having a travel range of $2.5 \mathrm{~cm}$ and a resolution of $5 \mu \mathrm{m}$. Additionally, a fourth stage was mounted on the mechatronic platform (8MR190-2-28, STANDA, Vilnius, Lithuania) in order to enable the rotation of the sample. Such stage had $360^{\circ}$ rotation range with $0.01^{\circ}$ resolution.

(ii) An ultrasound probe (Sonomed, mod. 2014059, Warsaw, Poland) with $16 \mathrm{MHz}$ central frequency and a fractional bandwidth equal to 0.25 at $-6 \mathrm{~dB}$ used in pulse-echo mode. The needle-type probe, $3 \mathrm{~mm}$ in diameter, was selected for directly contacting and indenting the sample. A $30 \mathrm{Vpp}$ pulsed excitation was delivered to the probe via a transmitter (US-Key, Lecoeur-Electronique, Chuelles, France) connected to a PC via USB2. The experimental setup was completed with the ultrasound data acquisition device, NI FlexRIO (National Instruments Corp., Austin, TX, USA), for acquisitions at high frequency $(1.6 \mathrm{GHz})$.

(iii) A load cell (Nano 43, ATI Industrial Automation, Apex, NC, USA) to collect interaction forces, up to $18 \mathrm{~N}$ with $0.004 \mathrm{~N}$ resolution along normal axis, arising at the interface between the ultrasound 
probe and the sample, also used in the control loop of the translation stages in order to operate force-controlled indentations. The developed software used this force data to calculate the stiffness and to trigger the high frequency US data collection at the threshold point of contact $(0.2 \mathrm{~N})$.

(iv) A waterproof HD-camera (Hero5 Session, GoPro, San Mateo, CA, USA) with $10 \mathrm{MP}$ and $4 \mathrm{~K}$ resolution, integrated to perform the sample shape recognition and to create a matrix of points to be indented.

(v) A stainless-steel disk fixed on the top of the motorized stages for the positioning of the sample, but also to permit the reflection of the ultrasound signal back to the probe. The disk had a diameter of $16 \mathrm{~cm}$ and a thickness of $1 \mathrm{~cm}$.

A

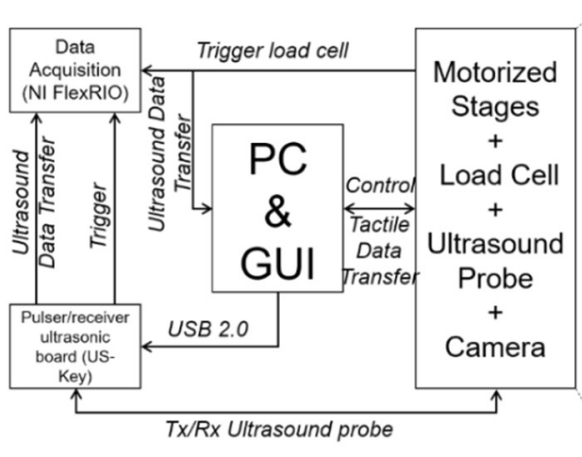

B

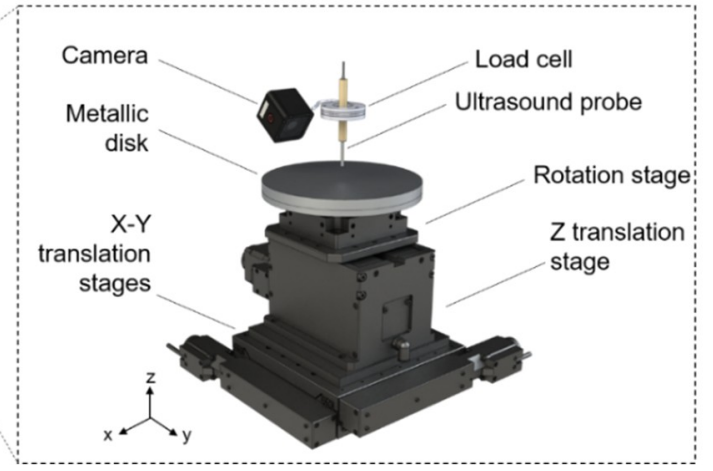

Figure 2. (A) Block diagram of the experimental setup. (B) Experimental setup showing the different components.

The software routines for controlling the platform and the automatic scan of the samples and for performing data acquisition, as well as the graphical user interfaces were developed in LabVIEW, LabVIEW Real-Time and LabVIEW FPGA (National Instruments Corp., Austin, TX, USA), while the data analyses were performed using MATLAB (The MathWorks, Inc., Natick, MA, USA).

\subsection{Phantom of Healthy Tissue and Inclusions}

Tests were performed on seven Agar block-shaped phantoms, realized to mimic both the mechanical and the acoustic properties of diseased human tissues. Each phantom had a soft surrounding matrix representing the human healthy tissue and hard inclusions embedded inside to represent tumor nodules. Each fabricated phantom was nominally $60 \mathrm{~mm}$ wide, $100 \mathrm{~mm}$ long and $15 \mathrm{~mm}$ thick, while the buried spherical inclusions had different diameters ranging from $3 \mathrm{~mm}$ to $12 \mathrm{~mm}$. The volume of the phantom was large enough to introduce up to 8 inclusions, 2 per each diameter, in different $X-Y$ positions with adequate separation distance (Figure 3 ) in order to execute computer-aided detection trials. It is worth to mention that the Agar phantom did not need any pre-treating before performing the automatic scan process. However, also in case of biological tissues, it is not necessary any further pre-treatments apart from the one required within custom histological evaluations.

Agar-based phantoms were prepared using a predefined concentration of Agar in distilled water. Changing the concentration of Agar resulted in a variation of both the mechanical and acoustic properties. A concentration of $2 \mathrm{~g}$ of Agar in $100 \mathrm{~mL}$ of water was used to represent a healthy human tissue (fabricating a phantom entirely with this concentration results in 1.59 MRayl acoustic impedance, $1457 \mathrm{~m} / \mathrm{s}$ speed of sound and $0.33 \mathrm{~N} / \mathrm{mm}$ mechanical impedance). A concentration of $8 \mathrm{~g}$ of Agar in $100 \mathrm{~mL}$ of water was used for simulating a tumor tissue (fabricating a phantom entirely with this concentration results in 1.92 MRayl acoustic impedance, $1534 \mathrm{~m} / \mathrm{s}$ speed of sound and $4.6 \mathrm{~N} / \mathrm{mm}$ mechanical impedance) [28,30-32]. 


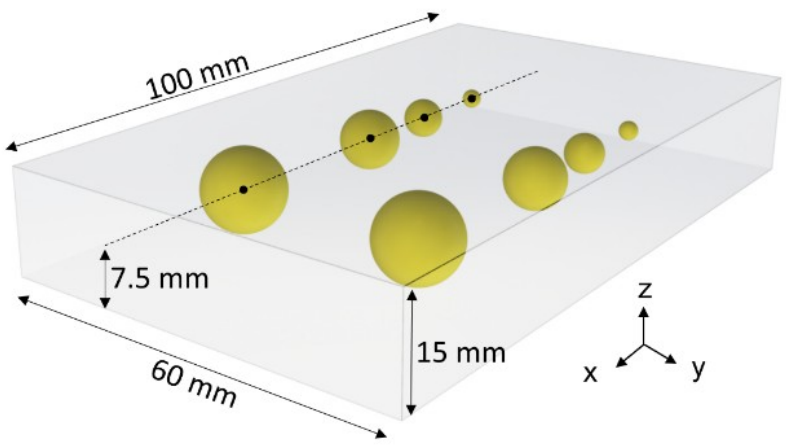

Figure 3. Rendering of the Agar phantom used during the experimental acquisition. The spherical inclusions are marked in yellow $(\emptyset 12-9-6-3 \mathrm{~mm})$. The volume of the phantom is $100 \times 60 \times 15 \mathrm{~mm}^{3}$.

\subsection{Experimental Protocol}

The experimental protocol consisted in an automatic scan of the sample. The procedure was divided in two steps:

(i) Visual analysis;

(ii) Stiffness and ultrasound analysis.

The purpose of the automatic visual analysis was to recognize the shape of the sample by acquiring its boundaries and to create the indentation matrix, namely the points to be analyzed. Such analysis is crucial when dealing with real tissues, where the shape and size is unknown or irregular, so that the scan can be defined automatically. The visual part (Figure 4) consisted in subtracting the background image from the sample image, thus obtaining the shape, the size and the orientation. Starting from this new image (Figure 4C), a set of indentation points was created with a $2 \mathrm{~mm}$ step along the $X$ and Y-axes.

A
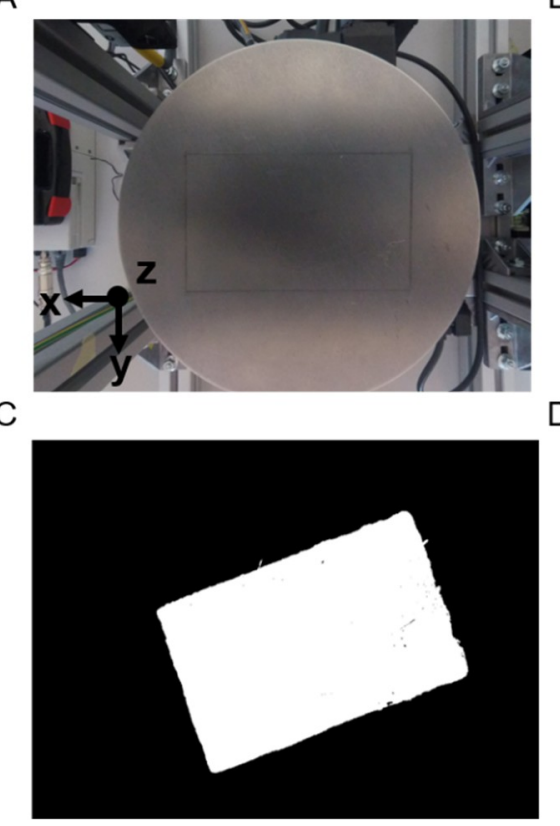

B
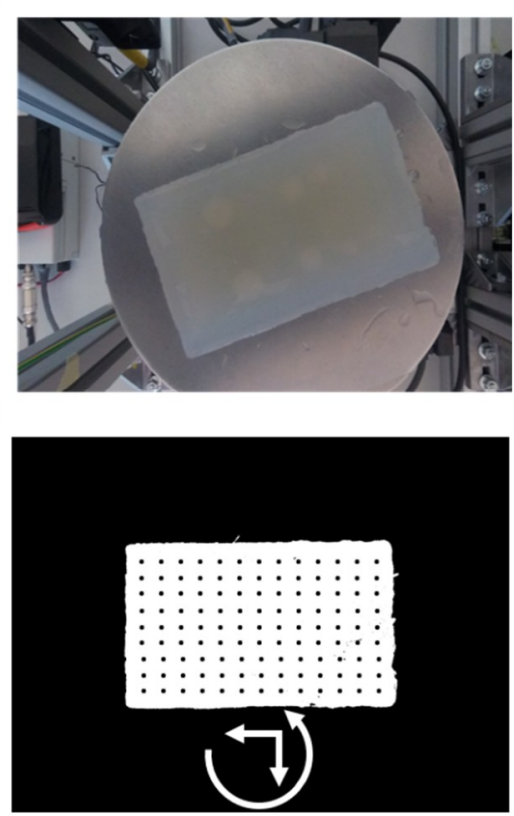

Figure 4. Visual part: Positioning of the sample, boundary detection and creation of the indentation matrix. (A) Background. (B) Sample in an arbitrary position. (C) Background subtraction. (D) Positioning by rotation of the sample and creation of the indentation matrix.

Once the visual analysis was completed, it was possible to start the acquisition of the compression force and ultrasound signals. Per each $X-Y$ point of the indentation matrix, the phantom was indented 
along the Z-axis at constant speed $(0.5 \mathrm{~mm} / \mathrm{s})$. The compression force was recorded and, at a low threshold $(0.2 \mathrm{~N}$, to avoid damaging the phantom), a trigger signal was generated for ultrasonic pulse transmission and reflected signal reception for recording (Figure 5). In a nutshell, the robotic platform control was fully automatic from the placement of the tissue onto the platform up to the localization of the inclusions. However, the system provided the user interface for an operator to supervise the scan according to the physician's requirements.

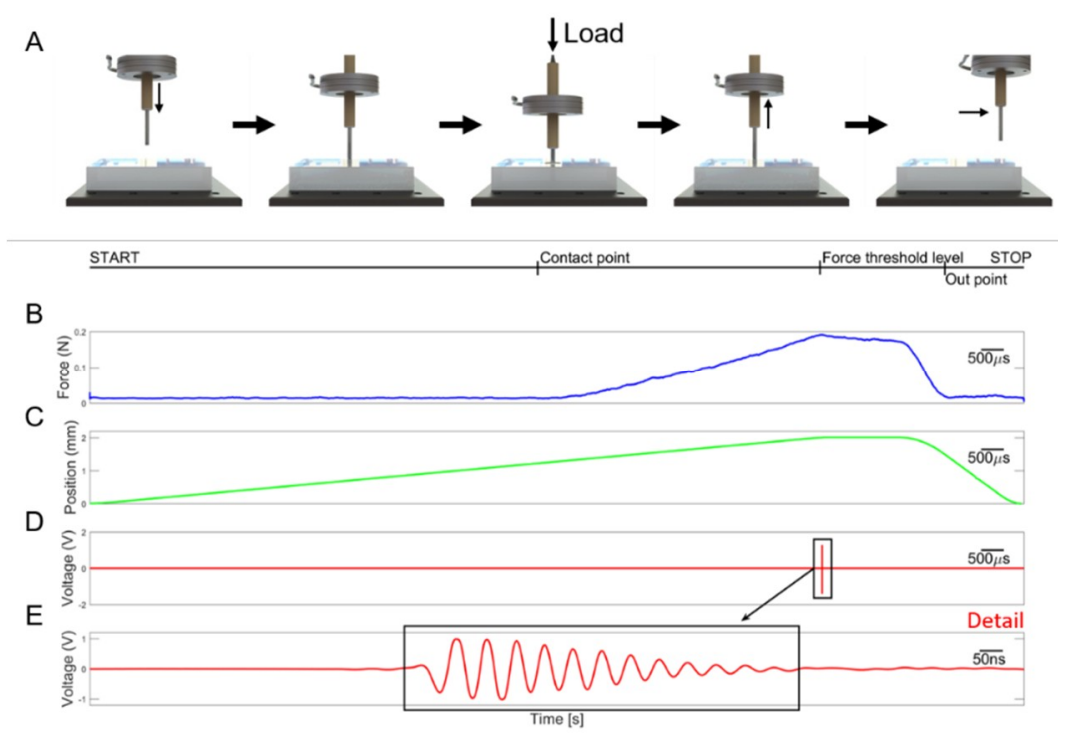

Figure 5. (A) Experimental protocol involving indentation of the ultrasound probe under regulation of the contact force. (B) Normal force. (C) Position along Z-axis. (D) Ultrasound signal reflected from the steel metal plate. (E) Zoom of the ultrasonic signal shown in panel D reflected at the tissue sample bottom in contact with the steel plate.

\subsection{Data Analysis}

The detection and localization of the different inclusions was based on the elaboration of indentation force $\left(F_{Z}\right)$, vertical position $(Z)$ and ultrasound signals. The stiffness parameter $k$ is the ratio between the change in force and the change in $Z$ displacement and for each indentation was calculated according to Equation (1). Here, $F_{z_{\max }}$ refers to the force threshold of the indentation and $F_{z_{0}}$ refers to force measured by the load cell following the first contact.

$$
k=\frac{\Delta F_{z}}{\Delta Z}=\frac{F_{z_{\max }}-F_{z_{0}}}{Z_{F_{z_{\max }}}-Z_{F_{z_{0}}}}
$$

The ultrasound technique used for the detection of the inclusions was based on the reflectometric method. In fact, we considered more reliable to work with the variation of the signal reflected from the interface created by the bottom of the phantom and the steel plate. This signal has a higher amplitude than the back scattered or reflected signal from the inclusion embedded into the tissue-like matrix. For our phantoms, according to the selected acoustic parameters mimicking healthy and cancerous tissues [31], we can estimate a reflection coefficient generated by the acoustic impedance difference at a planar interface less than $1 \%$. The ultrasonic analysis consisted in the processing of the signal detected in each point of the indentation matrix defining a Correlation Index Amplitude (CIA) parameter derived from [33], as reported in Equation (2):

$$
C I A=1-\left(\frac{\min \left(\sqrt{\sum S_{r e f}^{2}}, \sqrt{\sum S_{i}^{2}}\right)}{\max \left(\sqrt{\sum S_{r e f}^{2}}, \sqrt{\sum S_{i}^{2}}\right)}\right)
$$


In Equation (2), $S_{i}$ is the signal acquired in each point and $S_{r e f}$ is the reference signal. The CIA assumed values between 0 and 1: For two identical signals the CIA is zero, while for very different amplitude signals the CIA approaches to 1 . We assume that a reference signal can be acquired in a position where neither inclusions nor other inhomogeneity have been detected; under this assumption the transmitted signal from the US probe is only attenuated by the two-way travel path defined by the steel plate interface. Due to low tissue sample thickness (typically from $5 \mathrm{~mm}$ to $20 \mathrm{~mm}$ ) and moderate attenuation of the US at the operating frequency $(1.25 \mathrm{~dB} / \mathrm{cm} @ 16 \mathrm{MHz})$ this signal has an amplitude that is greater than the amplitude of the echo signals obtained in the probe positions over an inclusion; in our test samples, we found an amplitude variation of about $10 \%$ and $60 \%$ for the echo signal at the steel plate interface over and outside the inclusions, respectively. A high CIA indicates the detection of an inclusion since the two signals become poorly correlated.

For each indented point, a colour map was created both for stiffness and for correlation index amplitude. An unsupervised classifier, called Fuzzy C-mean (FCM) clustering [34], was used to classify each indentation of the scan on the phantom. Such unsupervised classification system, starting from the elaborated data, enabled the categorization of the point and the subsequent organization into different clusters. In this way, it was possible to divide the data into two classes: (a) Tumor class, which were the sites classified as inclusions, and (b) healthy class, which were the sites classified as non-inclusions. From the wrong classification prediction, we obtained the number of false positive, i.e., soft matrix points classified as inclusions, and the false negative number, i.e., inclusions classified as soft matrix. Furthermore, new datasets were obtained and classified by merging the stiffness and the ultrasound data using AND-OR logics. In the AND case, we considered tumor only the points identified as inclusion in both the datasets simultaneously, thus we expected an increase in the total number of false negatives. In the OR case, we considered tumor all the points classified as inclusion in either the stiffness dataset or the ultrasound dataset, thus we expected an increase of the number of false positives and reduced false negatives. The results of the OR logics are crucial to include all of the cancerous tissues. Through a confusion matrix, the accuracy and the misclassification rate were calculated for all the datasets and methods.

\section{Results}

All the experimental results presented in this section have been repeated over seven replicas of the developed phantoms.

\subsection{Results from Stiffness Measurements}

An elaboration example of the stiffness analysis, for one of the seven phantoms, is shown in the top parts of Figure 6. The bottom part of Figure 6A shows the positions of the inclusions inside the indentation matrix. Since the inclusions were embedded into a soft matrix, their stiffness was depending not only on the materials properties, like the elasticity, but also on their dimensions. The stiffness parameter recorded at the location of indentation is the complex homogenized combination of the inclusion below and the surrounding soft "healthy" tissue. Hence, the stiffness parameter, $k$ from Equation (1), was found to increase with the dimension. Stiffness analysis was clearly capable to detect the bigger inclusions, namely $12 \mathrm{~mm}$ and $9 \mathrm{~mm}$. Figure $6 \mathrm{~B}$, showing the results for the whole indentation matrix, confirmed this trend. A visual inspection of the image allows discriminating big inclusions compared to the soft surrounding matrix.

The results of the identification based on stiffness measurements are shown in Figure 7A, obtained by the Fuzzy C-mean (FCM) clustering. The results of this unsupervised classification system confirmed the ability of the stiffness measurement system to recognize all the points belonging to the big inclusions, thus without false negatives. Such performances were evident from the high number of true positive (green points) for $12 \mathrm{~mm}$ and $9 \mathrm{~mm}$ inclusions. However, stiffness analysis was not able to reliably identify the smallest inclusions, as pointed out by the high number of false negatives (red points) for $6 \mathrm{~mm}$ and $3 \mathrm{~mm}$ inclusions (Figure 7A). 
A

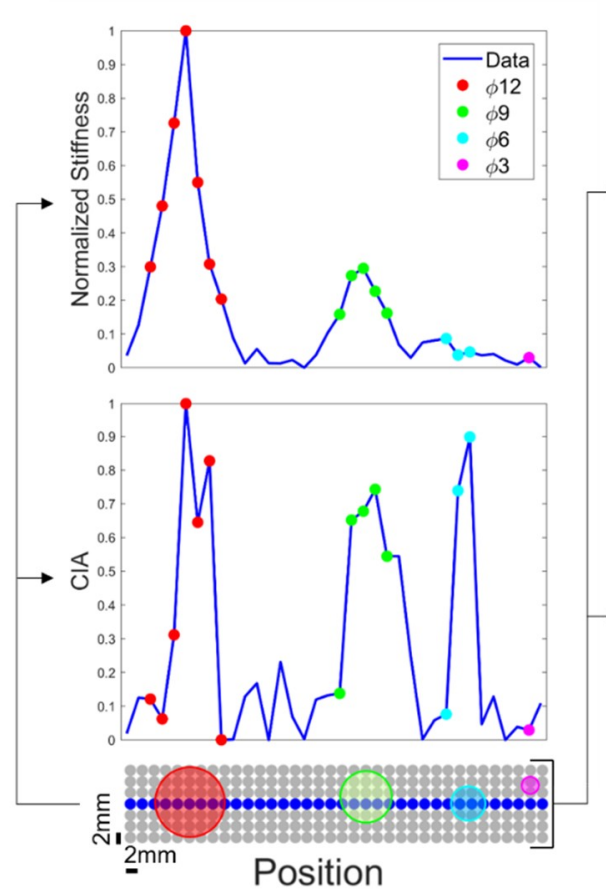

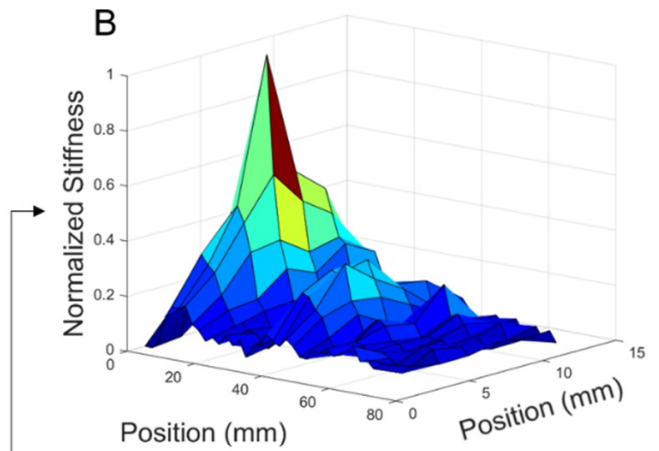

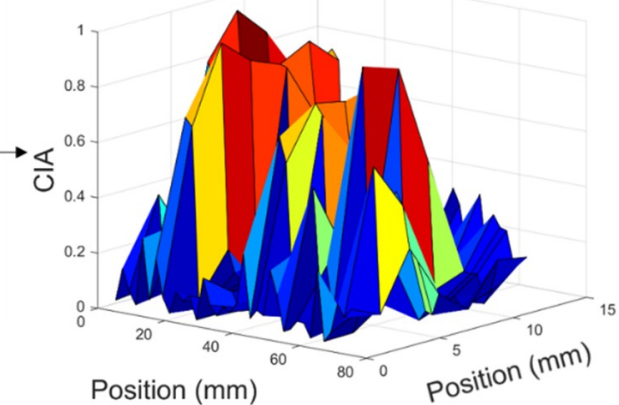

Figure 6. (A) (top) graph showing stiffness as a function of position, calculated as $\Delta \mathrm{Fz} / \Delta \mathrm{Z}$, for the central row; (bottom) graph showing ultrasound signal processing of CIA index. (B) (top) 3D graph showing stiffness across the whole indentation matrix; (bottom) 3D graph showing ultrasound signal processing of Correlation Index Amplitude (CIA) index.

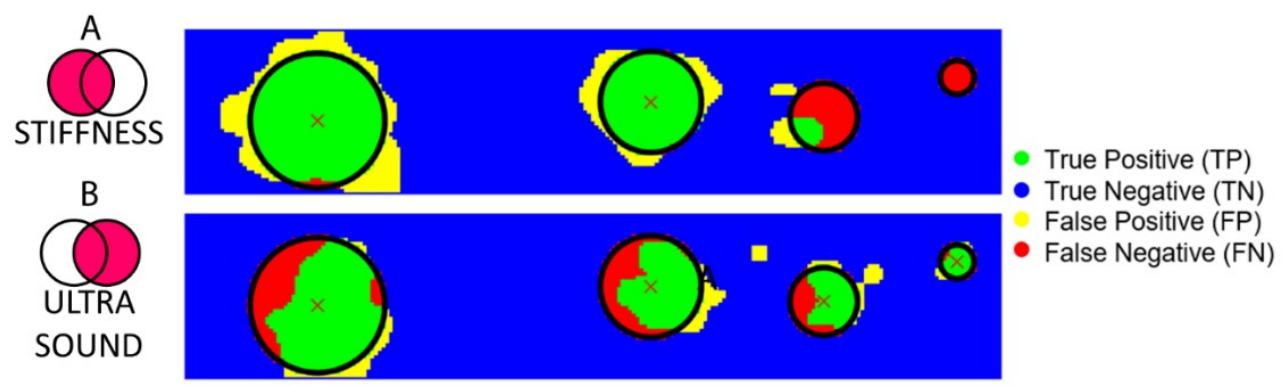

Figure 7. Classification (TP-TN-FP-FN) of all the points of the indentation matrix for the analyses with stiffness (A) and ultrasound (B) measurements.

\subsection{Results from Ultrasound Measurements}

According to the ultrasound data analysis, shown in Figure 6 (bottom part), we can observe in Figure 6A that the CIA index increases consistently in correspondence of the inclusions. However, unlike the stiffness measurements, higher CIA values were observed also for the smaller inclusions. Thanks to the high CIA peak recorded for each inclusion, this approach led to the detection of all the inclusions buried in the phantom. Figure $6 \mathrm{~B}$, showing the results for the whole indentation matrix, confirmed this trend. As for the stiffness measurement part, Figure 7B shows the results of the FCM clustering, highlighting the ability of the ultrasound system to detect each inclusion. The trend is visible in Figure 7B where true positives (in green) are present in each inclusion. Remarkably, false positives (in yellow) and false negatives (in red) were obtained in the area at the boundary between the inclusion and the soft matrix, confirming the high specificity in identifying the area to focus on for histological analyses. At such boundaries, the ultrasonic beam, coming from the source and returning to the source upon reflection on the stainless steel plate, could have experienced diffraction effects 
that produced an apparent enlargement of the real dimensions of the spherical inclusions thus giving origin to false positives.

\subsection{AND-OR Logics to Merge Stiffness and Ultrasound Measurements}

With the aim to improve the detection performance (true positives vs. false negatives), new datasets were obtained and classified by merging stiffness and ultrasound measurements using AND-OR logics and the corresponding results are shown in Figure 8. The AND logics (Figure 8A) turned out in an increase of false negatives and decrease of false positives. The growth of false negative predictions can lead to the worst-case scenario, since might bring to a loss of identified tumors. Instead, the OR logics demonstrated to be a safer approach since it turned out in an acceptable increase of false positives and a consistent decrease of false negatives. As shown in Figure 8B, the OR logics between stiffness and ultrasound measurements was able to correctly discriminate all the inclusions, even the smaller ones. Such results were achieved thanks to the complementarity of the two systems. It was observed that the stiffness analysis was better in localizing bigger inclusions, whereas the ultrasound analysis was better for the detection of smaller inclusions (compare Figure 7A,B).

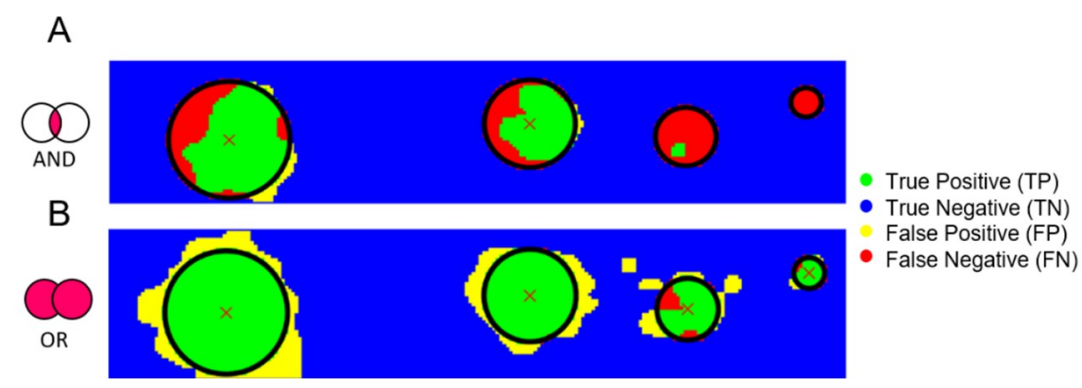

Figure 8. Classification (TP-TN-FP-FN) for all the points of the indentation matrix following the AND (A) and OR (B) logics of stiffness- and ultrasound-based classifications shown in Figure 7.

This behavior was further confirmed by the confusion matrices obtained with the seven experimented phantoms and with all the identification techniques, i.e., based on just stiffness measurements, just ultrasound, and with the AND-OR logics (Figure 9).
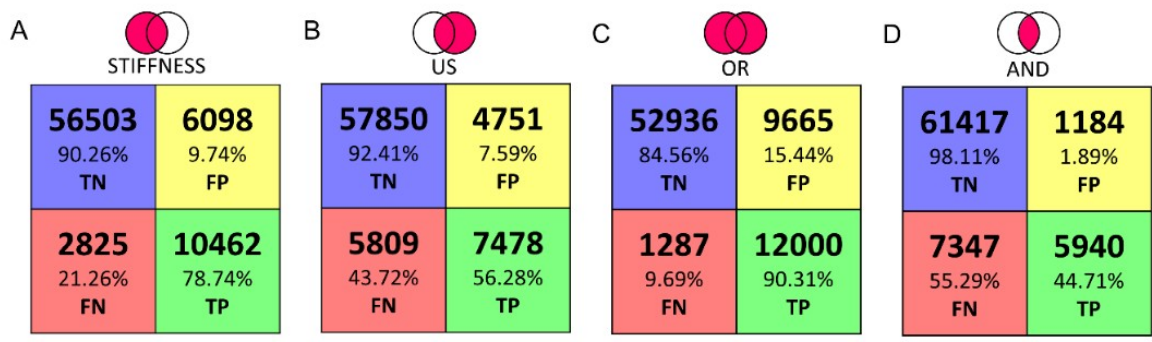

Figure 9. Confusion Matrix with classification based on (A) stiffness measurements; (B) ultrasound measurements; (C) stiffness or ultrasound measurements; (D) stiffness and ultrasound measurements.

\section{Discussion}

In this work we present a platform aiming at identifying cancer nodules in ex-vivo tissues. Such tool, oriented towards the automation of diagnostic procedures during surgery, has the scope of increasing the effectiveness of histopathological evaluations. Such exams need to be performed as correctly as possible because the report may lead in a modification of the surgical procedure. The human capability to detect these lumps with characteristic dimension of few $\mathrm{mm}$, depends on the pathologist expertise and tactile capabilities. To achieve this goal, the presented platform combines three different measurements, such as camera vision, stiffness calculations via force-position sensing 
and ultrasound recordings to perform an automatic scan and evaluation of the indented tissue. In this paper the tests were performed in a laboratory environment using seven Agar phantoms that mimicked the mechanical and acoustic properties of human ex-vivo tissues. The phantoms integrated eight spherical inclusions with different diameters (from $3 \mathrm{~mm}$ up to $12 \mathrm{~mm}$ ) to reproduce tumors inside healthy tissues. The results, for all phantoms, summarized in the confusion matrices, demonstrated the ability of the platform to automatically identify the inclusions, particularly when complementing stiffness with ultrasound measurements via OR logics. In particular, as reported in the confusion matrix, the tactile analysis presents valuable classification results in detecting the inclusions as reflected from the $78.73 \%$ of TP and $90.26 \%$ of TN. Moreover, it shows a low percentage of FP and FN, $9.74 \%$ and $21.27 \%$, respectively. We observe that the tactile analysis provides satisfactory shape recognition and tumor detection for inclusions above $6 \mathrm{~mm}$ in diameter. On the other hand, it missed the smaller inclusions that were buried deeper into the softer matrix. The ultrasound analysis can be a very good guiding tool for localization and detection of tumors, including the smaller ones, because the ultrasound resolution is much higher than the size of the inclusion and the difference in the acoustic impedance along the $z$-axis is sufficient to generate an amplitude variation than can be detected from noise. The ultrasound data presents high amount of TN of $92.41 \%$ and a low FP of $7.59 \%$. However, the ultrasound alone shows a high number of FN of $43.72 \%$. To improve the performance, the classified datasets were logically merged using the OR and AND logics. As expected, the results of OR logics gave evidence of a higher rate of inclusions recognition (i.e., $90.3 \%$ of TP and $84.56 \% \mathrm{TN}$ ), while maintaining low error rates (i.e., $9.68 \% \mathrm{FN}$ and $15.44 \% \mathrm{FP}$ ). Such a result is a direct consequence of the implementation of this logics, since we considered all the points classified as inclusion, in either the stiffness dataset or the ultrasound dataset, as tumor. This entails a better localization and reconstruction of the buried inclusions. Interestingly, the AND logics localizes the bigger inclusions with an increased TN rate of $98.10 \%$ and reduced the FP rate to $1.90 \%$, but the TP rate of $44.70 \%$ and FN rate of $55.30 \%$ missed the correct shape and smaller tumors entirely.

In addition, we found that the ultrasound method was also sensitive to the presence of air bubbles formed in the agar inclusions as the surface of such bubbles might introduce a significant impedance contrast for the ultrasound signals. This could create amplitude variation in the reflected signal that resembled to the "healthy tissue". Thanks to the good spatial resolution of our system, the positions around the air bubbles provide faithful data that can reduce the impact of this constraint; moreover, the biological tissues are expected to not have air bubbles. However, the tactile data were not sensitive to these air bubbles inside the inclusions, reproducing their shape a more faithfully in the OR logics. Within the present work, we adopted a scan resolution with step of $2 \mathrm{~mm}$ inspired by the $16 \mathrm{MHz}$ needle probe diameter (i.e., $3 \mathrm{~mm}$ ). To keep a balance between the scan speed and area, we decided to scan with step of $2 \mathrm{~mm}$. Lesser resolution values lead to insufficient data points in the scanned area, while higher values would introduce unaffordable scan time and oversampling.

The phantoms we used were the simplistic versions of the biological tissues. Hence, further developments will address the experimentation of the robotic platform on ex-vivo tissues. After this validation step, we can envisage that the sensorized platform placed in the operating theatre will enable the pathologist to access data remotely with the purpose of assisting the surgeon in adapting the procedures during surgery. Information obtained from the platform can also be used to provide haptic feedback to the pathologist by means of wearable interfaces [35-38]. The analysis of vision data, now used only for detecting the boundary of the tissue and thus to define the indentation matrix, can be improved to provide a visual report too. Such a new procedure will target the extraction of several features from the pictures of both healthy and tumorous tissues to learn their differences via artificial intelligence methods and thus complement stiffness and ultrasound measurements. Finally, the results will be translated in an electronic report and integrated with the management software (e.g., HL7) of the healthcare system. 
Author Contributions: L.M. (Luca Massari) developed the mechatronic platform, integrated the experimental setup, co-designed the experimental protocol, performed the experimental protocol, analyzed the data, discussed the results and edited the paper; A.B. realized the phantom, co-designed the experimental protocol, performed the experimental protocol, analyzed the data, discussed the results and edited the paper; S.P. realized the phantom, co-designed the experimental protocol, performed the experimental protocol, analyzed the data, discussed the results and edited the paper; M.M. realized the phantom, co-designed the experimental protocol, performed the experimental protocol, discussed the results; F.F. handled the project administration, discussed the results and revised the paper; E.V. analyzed the data and discussed the results; M.P. developed the visual analysis and discussed the results; F.S., F.C., L.M. (Luca Messerini), provided medical background, co-designed the experimental protocol, contributed to perform the experimental protocol and revised the paper; G.C. co-supervised the development of the mechatronic platform, co-designed the experimental protocol and revised the paper; L.C. and A.M. co-supervised the development of the mechatronic platform, co-designed the experimental protocol, contributed to data analysis, discussed the results and revised the paper; C.M.O. designed and supervised the study, supervised the development of the mechatronic platform, co-designed the experimental protocol, contributed to data analysis, discussed the results and revised the paper.

Funding: This work was supported in part by the Tuscany Region within the FAS-Salute call, via the IMEROS project (CUPD66D16000120002).

Acknowledgments: This paper is a result of a collaborative project involving different institutes and entities. The authors thank all the collaborators for their valuable contributions.

Conflicts of Interest: The authors submitted a patent on the platform presented in the present study. The funders had no role in the design of the study; in the collection, analyses, or interpretation for data; in the writing of the manuscript, or in the decision to publish the results.

\section{References}

1. Pierangelo, A.; Benali, A.; Antonelli, M.-R.; Novikova, T.; Validire, P.; Gayet, B.; Martino, A. De Ex-vivo characterization of human colon cancer by Mueller polarimetric imaging. Opt. Express 2011, $19,1582$. [CrossRef] [PubMed]

2. Adam, R. Chemotherapy and surgery: New perspectives on the treatment of unresectable liver metastases. Ann. Oncol. 2003, 14, ii13-ii16. [CrossRef] [PubMed]

3. Nagai, T.; Niikura, H.; Okamoto, S.; Nakabayashi, K.; Matoda, M.; Utsunomiya, H.; Nagase, S.; Watanabe, M.; Takeshima, N.; Yaegashi, N. A new diagnostic method for rapid detection of lymph node metastases using a one-step nucleic acid amplification (OSNA) assay in endometrial cancer. Ann. Surg. Oncol. 2015, 22, 980-986. [CrossRef] [PubMed]

4. Candefjord, S.; Ramser, K.; Lindahl, O.A. Technologies for localization and diagnosis of prostate cancer. J. Med. Eng. Technol. 2009, 33, 585-603. [CrossRef] [PubMed]

5. Krouskop, T.A.; Wheeler, T.M.; Kallel, F.; Garra, B.S.; Hall, T. Elastic moduli of breast and prostate tissues under compression. Ultrason. Imaging 1998, 20, 260-274. [CrossRef] [PubMed]

6. Samani, A.; Zubovits, J.; Plewes, D. Elastic moduli of normal and pathological human breast tissues: An inversion-technique-based investigation of 169 samples. Phys. Med. Biol. 2007, 52, 1565. [CrossRef] [PubMed]

7. Zhang, M.; Nigwekar, P.; Castaneda, B.; Hoyt, K.; Joseph, J.V.; di Sant'Agnese, A.; Messing, E.M.; Strang, J.G.; Rubens, D.J.; Parker, K.J. Quantitative characterization of viscoelastic properties of human prostate correlated with histology. Ultrasound Med. Biol. 2008, 34, 1033-1042. [CrossRef]

8. Raveh Tilleman, T.; Tilleman, M.M.; Neumann, H.A.M. The elastic properties of cancerous skin: Poisson's ratio and Young's modulus. Isr. Med. J. 2004, 6, 753-755.

9. Winstone, B.; Melhuish, C.; Pipe, T.; Callaway, M.; Dogramadzi, S. Toward Bio-Inspired Tactile Sensing Capsule Endoscopy for Detection of Submucosal Tumors. IEEE Sens. J. 2017, 17, 848-857. [CrossRef]

10. Carter, F.J.; Frank, T.G.; Davies, P.J.; McLean, D.; Cuschieri, A. Measurements and modelling of the compliance of human and porcine organs. Med. Image Anal. 2001, 5, 231-236. [CrossRef]

11. Konstantinova, J.; Li, M.; Mehra, G.; Dasgupta, P.; Althoefer, K.; Nanayakkara, T. Behavioral characteristics of manual palpation to localize hard nodules in soft tissues. IEEE Trans. Biomed. Eng. 2014, 61, 1651-1659. [CrossRef] [PubMed]

12. Wells, P.N.T.; Liang, H.H.-D. Medical ultrasound: Imaging of soft tissue strain and elasticity. J. R. Soc. Interface 2011, 8, 1521-1549. [CrossRef] [PubMed] 
13. Carson, W.C.; Gerling, G.J.; Krupski, T.L.; Association Kowalik, C.G.; Harper, J.C.; Moskaluk, C.A. Material characterization of ex vivo prostate tissue via spherical indentation in the clinic. Med. Eng. Phys. 2011, 33, 302-309. [CrossRef] [PubMed]

14. Gwilliam, J.C.; Yoshioka, T.; Okamura, A.M.; Hsiao, S.S. Neural coding of passive lump detection in compliant artificial tissue. J. Neurophysiol. 2014, 112, 1131-1141. [CrossRef] [PubMed]

15. Yau, J.M.; Kim, S.S.; Thakur, P.H.; Bensmaia, S.J. Feeling form: The neural basis of haptic shape perception. J. Neurophysiol. 2016, 115, 631-642. [CrossRef] [PubMed]

16. Nam, K.; Rosado-Mendez, I.M.; Wirtzfeld, L.A.; Pawlicki, A.D.; Kumar, V.; Madsen, E.L.; Ghoshal, G.; Lavarello, R.J.; Oelze, M.L.; Bigelow, T.A. Ultrasonic attenuation and backscatter coefficient estimates of rodent-tumor-mimicking structures: Comparison of results among clinical scanners. Ultrason. Imaging 2011, 33, 233-250. [CrossRef]

17. Hoch, G.; Croise-Laurent, V.; Germain, A.; Brunaud, L.; Bresler, L.; Ayav, A. Is intraoperative ultrasound still useful for the detection of colorectal cancer liver metastases? Int. Hepato Pancreato Biliary Assoc. 2015, 17, 514-519. [CrossRef] [PubMed]

18. Hata, S.; Imamura, H.; Aoki, T.; Hashimoto, T.; Akahane, M.; Hasegawa, K.; Bekku, Y.; Sugawara, Y.; Makuuchi, M.; Kokudo, N. Value of visual inspection, bimanual palpation, and intraoperative ultrasonography during hepatic resection for liver metastases of colorectal carcinoma. World J. Surg. 2011, 35, 2779-2787. [CrossRef]

19. Ferrero, A.; Langella, S.; Giuliante, F.; Viganò, L.; Vellone, M.; Zimmitti, G.; Ardito, F.; Nuzzo, G.; Capussotti, L. Intraoperative liver ultrasound still affects surgical strategy for patients with colorectal metastases in the modern era. World J. Surg. 2013, 37, 2655-2663. [CrossRef] [PubMed]

20. Chou, R.; Cuevas, C.; Fu, R.; Devine, B.; Wasson, N.; Ginsburg, A.; Zakher, B.; Pappas, M.; Graham, E.; Sullivan, S.D. Imaging techniques for the diagnosis of hepatocellular carcinoma: A systematic review and meta-analysis. Ann. Intern. Med. 2015, 162, 697-711. [CrossRef] [PubMed]

21. Sigrist, R.M.S.; Liau, J.; El Kaffas, A.; Chammas, M.C.; Willmann, J.K. Ultrasound elastography: Review of techniques and clinical applications. Theranostics 2017, 7, 1303. [CrossRef] [PubMed]

22. Baker, A.R.; Windsor, C.G. The classification of defects from ultrasonic data using neural networks: The Hopfield method. NDT Int. 1989, 22, 97-105. [CrossRef]

23. Jain, A.K. Data clustering: 50 years beyond K-means. Pattern Recognit. Lett. 2010, 31, 651-666. [CrossRef]

24. Ahn, B.M.; Kim, J.; Ian, L.; Rha, K.H.; Kim, H.J. Mechanical property characterization of prostate cancer using a minimally motorized indenter in an ex vivo indentation experiment. Urology 2010, 76, 1007-1011. [CrossRef] [PubMed]

25. Barr, R.G.; Ferraioli, G.; Palmeri, M.L.; Goodman, Z.D.; Garcia-Tsao, G.; Rubin, J.; Garra, B.; Myers, R.P.; Wilson, S.R.; Rubens, D. Elastography assessment of liver fibrosis: Society of radiologists in ultrasound consensus conference statement. Radiology 2015, 276, 845-861. [CrossRef] [PubMed]

26. Li, W.; Belmont, B.; Greve, J.M.; Manders, A.B.; Downey, B.C.; Zhang, X.; Xu, Z.; Guo, D.; Shih, A. Polyvinyl chloride as a multimodal tissue-mimicking material with tuned mechanical and medical imaging properties. Med. Phys. 2016, 43, 5577-5592. [CrossRef]

27. Culjat, M.O.; Goldenberg, D.; Tewari, P.; Singh, R.S. A review of tissue substitutes for ultrasound imaging. Ultrasound Med. Biol. 2010, 36, 861-873. [CrossRef] [PubMed]

28. Cafarelli, A.; Verbeni, A.; Poliziani, A.; Dario, P.; Menciassi, A.; Ricotti, L. Tuning acoustic and mechanical properties of materials for ultrasound phantoms and smart substrates for cell cultures. Acta Biomater. 2017, 49, 368-378. [CrossRef] [PubMed]

29. Cao, R.; Huang, Z.; Varghese, T.; Nabi, G. Tissue mimicking materials for the detection of prostate cancer using shear wave elastography: A validation study. Med. Phys. 2013, 40, 022903. [CrossRef] [PubMed]

30. Manickam, K.; Machireddy, R.R.; Seshadri, S. Study of ultrasound stiffness imaging methods using tissue mimicking phantoms. Ultrasonics 2014, 54, 621-631. [CrossRef]

31. Bamber, J.C.; Hill, C.R. Acoustic properties of normal and cancerous human liver-I. Dependence on pathological condition. Ultrasound Med. Biol. 1981, 7, 121-133. [CrossRef]

32. Azhari, H. Basics of Biomedical Ultrasound for Engineers; John Wiley \& Sons: Hoboken, NJ, USA, 2010.

33. Bulletti, A.; Giannelli, P.; Calzolai, M.; Capineri, L. An integrated acousto/ultrasonic structural health monitoring system for composite pressure vessels. IEEE Trans. Ultrason. Ferroelectr. Freq. Control 2016, 63, 864-873. [CrossRef] [PubMed] 
34. Bezdek, J.C.; Ehrlich, R.; Full, W. FCM: The fuzzy c-means clustering algorithm. Comput. Geosci. 1984, 10, 191-203. [CrossRef]

35. Sorgini, F.; Massari, L.; D'Abbraccio, J.; Palermo, E.; Menciassi, A.; Petrovic, P.; Mazzoni, A.; Carrozza, M.; Newell, F.; Oddo, C. Neuromorphic Vibrotactile Stimulation of Fingertips for Encoding Object Stiffness in Telepresence Sensory Substitution and Augmentation Applications. Sensors 2018, 18, 261. [CrossRef] [PubMed]

36. Massari, L.; D’Abbraccio, J.; Baldini, L.; Sorgini, F.; Farulla, G.A.; Petrovic, P.; Palermo, E.; Oddo, C.M. Neuromorphic haptic glove and platform with gestural control for tactile sensory feedback in medical telepresence applications. In Proceedings of the 2018 IEEE International Symposium on Medical Measurements and Applications (MeMeA), Rome, Italy, 11-13 June 2018; pp. 1-6.

37. Sorgini, F.; Mazzoni, A.; Massari, L.; Caliò, R.; Galassi, C.; Kukreja, S.; Sinibaldi, E.; Carrozza, M.; Oddo, C. Encapsulation of Piezoelectric Transducers for Sensory Augmentation and Substitution with Wearable Haptic Devices. Micromachines 2017, 8, 270. [CrossRef] [PubMed]

38. D'Abbraccio, J.; Massari, L.; Prasanna, S.; Baldini, L.; Sorgini, F.; Airò Farulla, G.; Bulletti, A.; Mazzoni, M.; Capineri, L.; Menciassi, A. Haptic Glove and Platform with Gestural Control For Neuromorphic Tactile Sensory Feedback In Medical Telepresence. Sensors 2019, 19, 641. [CrossRef] [PubMed]

(C) 2019 by the authors. Licensee MDPI, Basel, Switzerland. This article is an open access article distributed under the terms and conditions of the Creative Commons Attribution (CC BY) license (http://creativecommons.org/licenses/by/4.0/). 